\title{
The application of a modified neuroprosthetic hand system in a child with a C7 spinal cord injury. Case report
}

\author{
B T Smith MS, M J Mulcahey MS OTR/L, R J Triolo PhD, R R Betz MD \\ Functional Neuromuscular Stimulation Laboratory, Shriners Hospitals for Crippled \\ Children, 8400 Roosevelt Blvd, Philadelphia, PA 19152, USA.
}

\begin{abstract}
A neuroprosthetic hand system developed at Case Western Reserve University has been modified for use by an 8 year old child with an incomplete $\mathrm{C} 7$ spinal cord injury. This system has been adapted to accommodate voluntary thumb and finger extension, and provides stimulated finger flexion and thumb position for lateral and palmar prehension. Three months were required to develop grasps with sufficient strength and coordination for functional use. This period consisted of: implantation and immobilization of percutaneous intramuscular electrodes; stimulated exercise of the muscles of the hand and forearm; programming grasp patterns; and system training. Functional assessments show that the neuroprosthetic hand system allows the subject to perform unilateral and bilateral tasks that were otherwise impossible or were previously performed bimanually. The ability to perform activities of daily living with one hand frees the contralateral upper extremity to be used either for balance which increases the work area, or to stabilize an object allowing manipulation with the instrumented hand. Telephone interviews suggest that the hand system is used on a consistent basis at home and school. This single subject application indicates that a stimulation system designed for adults with C5-6 spinal cord injuries can enhance hand function and facilitate independence in a child with a low level cervical lesion.
\end{abstract}

Key words: functional neuromuscular stimulation; spinal cord injury; pediatrics; quadriplegia; neuroprosthetic hand system.

\section{Introduction}

In January 1989 the Philadelphia Unit of Shriners Hospitals for Crippled Children began a functional neuromuscular stimulation (FNS) research program in conjunction with Case Western Reserve University (CWRU). The project is part of a multicenter study to evaluate the relative costs and benefits of FNS technology for restoring active grasp to people with C5 and C6 spinal cord injuries. Their latest generation FNS system has been transferred to Shriners Hospital and adapted for use with children and adolescents with spinal cord injuries.

This paper reports on the application of the hand system to an eight year old child

Correspondence: Brian T. Smith, FNS Laboratory, Shriners Hospitals for Crippled Children, 8400 Roosevelt Blvd., Philadelphia, PA 19152, U.S.A. with a $\mathrm{C} 7$ level injury. ${ }^{1}$ Implementation and system training techniques are reported as well as preliminary results in which function with the FNS system is compared to unassisted function.

\section{Background}

For the past 12 years, researchers at CWRU have been studying FNS as a means of restoring lateral and palmar prehension to adults with spinal cord injuries at the fifth and sixth cervical level. ${ }^{2-7}$ Their FNS system consists of 3 major components: percutaneous intramuscular fine wire electrodes, a microprocessor-controlled stimulator, and a shoulder position sensor for command input.

Electrodes are implanted from common sites on the volar and dorsal forearm and are tunneled subcutaneously to forearm and 
thumb muscles. The electrodes are connected to a multichannel stimulator which generates the electrical current at programmed frequencies and pulse durations.

A shoulder position transducer allows stimulation to be controlled with the voluntary movement of the contralateral shoulder. A quick shoulder shrug locks the grasp in the desired amount of flexion. A second quick jerk returns control of stimulation to the user. A switch adjacent to the transducer allows the user to choose a grasp pattern and turn the system on and off. Corresponding audio cues indicate the system mode. ${ }^{5,6}$

The earliest reports of functional benefits obtained with the FNS system consisted of qualitative observation and interviews with system users. $^{7}$ In 1988, CWRU developed an evaluation to quantify the proficiency and consistency of hand grasp performance using the FNS system. This assessment consists of pinch and grip force measurements and unilateral manipulation of objects which are analogous to those used during activities of daily living (ADLs). Three objects are prehended with the palmar grasp (can, videotape, block) and 3 with the lateral (weight, peg, fork). For a given subject, consistency between evaluations can be measured and comparisons can be made between the FNS system and natural tenodesis or passive hand function. ${ }^{8}$

\section{Clinical profile}

An eight year old child at birth was found to have a hyperextension injury of his spine at the fifth and sixth cervical level. Table I and Test 1 in Table II show the left upper extremity manual muscle test ${ }^{9,10}$ results prior to FNS

Table I Left upper extremity muscle grades prior to FNS participation

\begin{tabular}{lc}
\hline Muscle & Grade \\
\hline Bicep & 5 \\
Brachioradialis & 5 \\
Tricep & 5 \\
Supinator & 5 \\
Pronator teres & 5 \\
FCR & 5 \\
FCU & 5 \\
PL & Absent \\
ECRL & 5 \\
ECRB & 5 \\
ECU & 5 \\
\hline
\end{tabular}

Table II Left hand muscle grades. Test 1 was performed before FNS implementation and Tests 2-4 were performed at specific points during FNS implementation. An asterisk denotes muscles which increased in grade.

\begin{tabular}{lcccc}
\hline Muscle & Test 1 & Test 2 & Test 3 & Test 4 \\
\hline FDPi & 0 & 0 & 0 & 0 \\
FDPl & $1+$ & $1+$ & $1+$ & $1+$ \\
FDPr* & 1 & 1 & 1 & 2 \\
FDPs & 1 & 1 & 1 & 1 \\
FDSi & 1 & 1 & 1 & 1 \\
FDS1 & 1 & 2 & 2 & 2 \\
FDSr* & 1 & 2 & 2 & 2 \\
FDSs & 1 & 1 & 1 & 1 \\
Lumbricales* & 2 & 4 & 4 & 4 \\
FPL & 1 & 1 & 1 & 1 \\
FPB & 1 & 1 & 1 & 1 \\
EDC* & 3 & 4 & 4 & 4 \\
EPL & 3 & 3 & 3 & 3 \\
EPB & 3 & 3 & 3 & 3 \\
AdP & 3 & 3 & 3 & 3 \\
AbPL* & 3 & 3 & 5 & 5 \\
OP & 0 & 0 & 0 & 0 \\
Interossei & 0 & 0 & 0 & 0 \\
\hline
\end{tabular}


participation. The patient has upper extremity motor sparing up to and including the $\mathrm{C} 7-\mathrm{C} 8$ level and is classified as a Frankel $\mathrm{C}$. He has a total motor index of 38 and according to the International Classification for Hand Reconstruction, ${ }^{11}$ he is classified as an $\mathrm{O}(\mathrm{CU}): 5$. Full passive range of motion in both hands is achieved with minimal hypermobility. Sensation bilaterally is intact to proprioception, temperature, stereognosis, and sharp touch.

The child has compensated for lack of hand function. Most play activities are performed bimanually with the palms providing the majority of the force to sustain grasps of objects (Fig 1). Because both upper extremities are engaged during performance of activities and unable to assist with balance, the work area is limited to arm's length at midline. To grasp small toys he positions objects between his index and middle fingers of his left hand and extends (Fig 2). He uses 2 hands to maintain utensils, manipulate his toothbrush, and write with a pencil (Fig 3). As a result of his bimanual prehension, he has difficulty keeping up with his able bodied peers

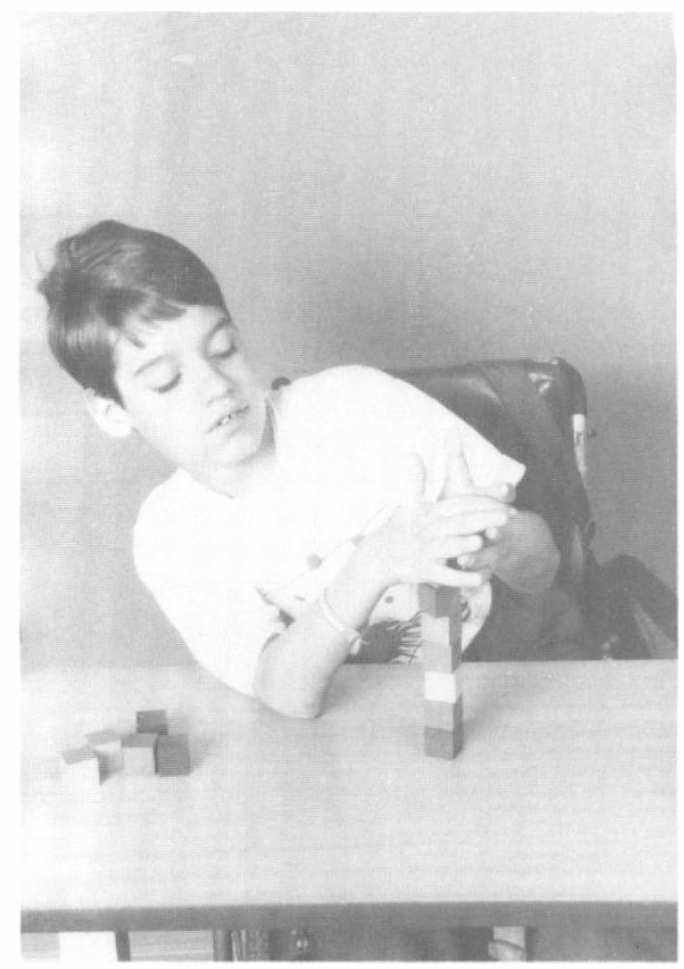

Figure 1 Bimanual function prior to FNS participation. His palms provide the force to sustain grasp of objects. Note the limited work space and poor trunk stability.

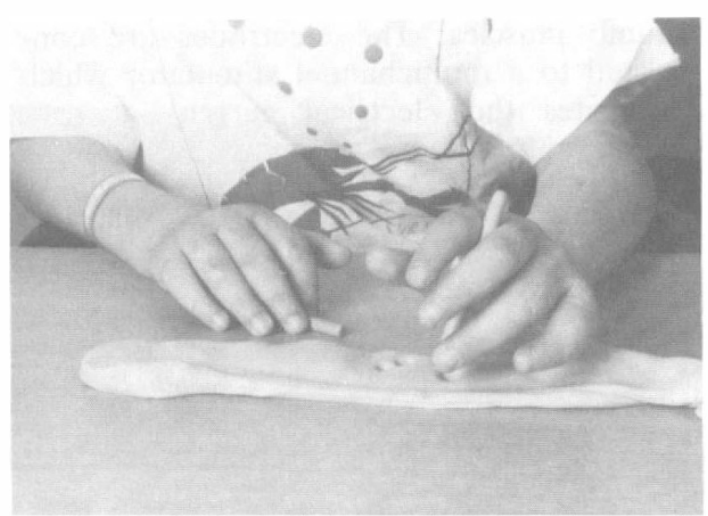

Figure 2 The child manipulates small objects by extension of the index and middle fingers.

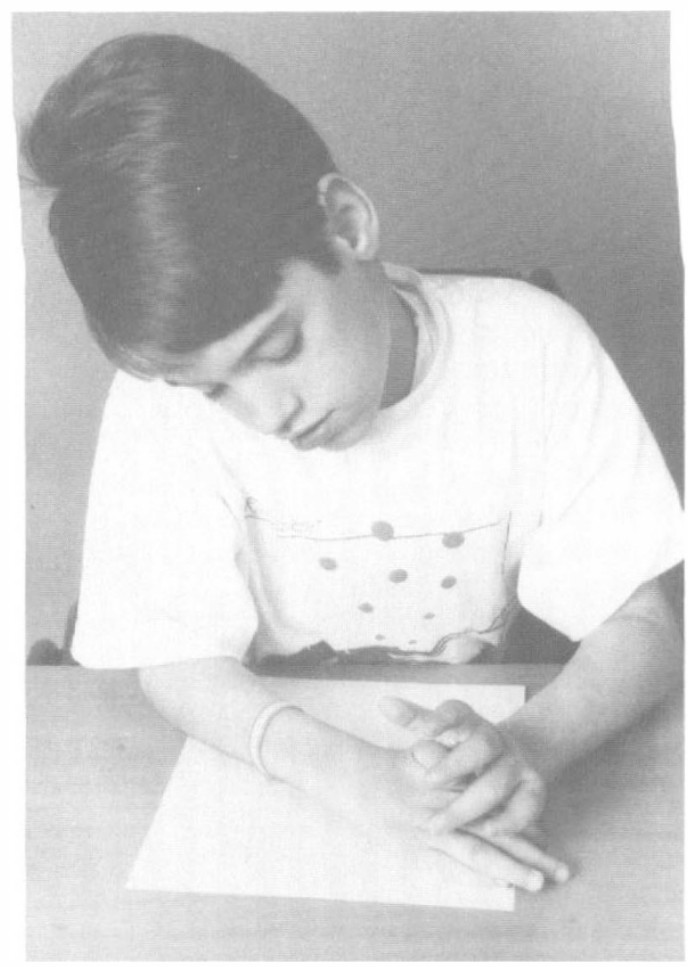

Figure 3 Typical prehension pattern for writing activities without FNS.

during in-class writing assignments and often requires excessive time to complete written homework assignments. His compensatory hand function does not enable him to assist in catheterization, write on the board at school, zipper a jacket, or use a knife. 
Although the subject did not meet the inclusion criteria of a complete C5-C6 injury level as established by CWRU, 12 it was felt that a modified FNS system would enhance his hand function and facilitate a global improvement in his ability to perform play, school, and self care activities.

\section{Implementation}

The patient was deemed appropriate for the FNS program after undergoing physiological, psychosocial, and environmental evaluations developed and administered by a multidisciplinary team of physical, recreational, and occupational therapists, biomedical engineers, social workers, nurses, and an orthopedic surgeon.

\section{Grasp patterns}

Figure 4 is a timeline depicting the duration and sequence of events necessary to instrument the left upper extremity with a modified CWRU neuroprosthetic hand system that provides finger flexion and proper thumb position for lateral and palmar grasps. Because of his age and intact sensation, implantation was under general anesthesia. Electrodes were implanted in the flexor digitorum superficialis (FDS) and flexor digitorum profundus (FDP) to flex the fingers in both grasps, in the adductor pollicis (AdP) for thumb adduction in the lat- eral grasp, flexor pollicis brevis (FPB), and flexor pollicis longus (FPL) for thumb flexion in the lateral grasp, and in the abductor pollicis brevis (AbPB) for thumb abduction in the palmar grasp.

Electrodes were implanted percutaneously using one of two methods. Most often, an electrode was implanted at the motor point of a specific muscle and then passed subcutaneously to a common exit site on the volar side of the forearm. With the second technique, an electrode was guided subcutaneously from the common volar exit site to the desired motor point where it was inserted into the muscle belly.

As Figure 4 shows, after the first implant the left upper extremity was immobilized in the resting hand position for 2 weeks in a short arm cast to promote healing and electrode encapsulation. After immobilization, the muscle responses were profiled, ${ }^{5}$ exercise patterns were established and the muscles were exercised by stimulation 6 hours a day, 6 days a week for 4 weeks to improve muscle strength and endurance. Stimulation was achieved using a constant current, charge-balanced biphasic waveform at pulse durations of up to 200 microseconds and a stimulus period of 60 milliseconds.

After the initial exercise period, several electrodes were found to be broken, necessitating a second implant and 2 week immobilization period. During the second implant, broken electrodes were replaced and backup electrodes were implanted. Due to some muscle atrophy

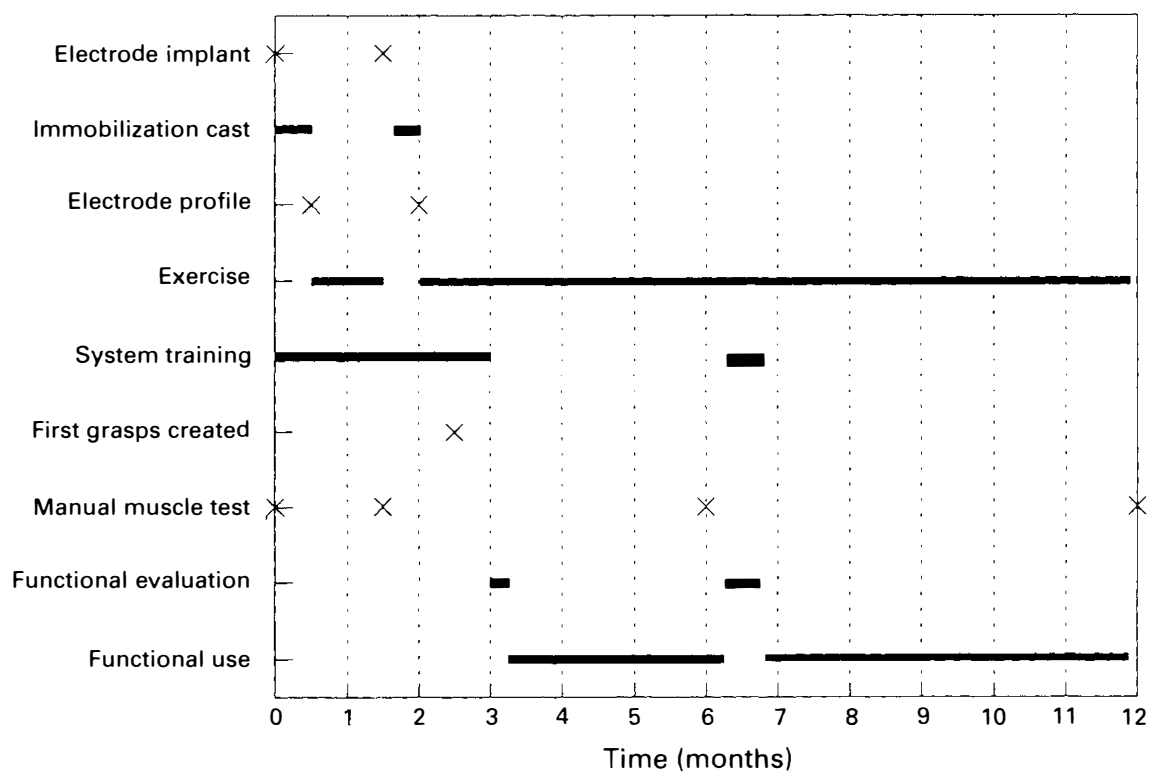

Figure 4 Timeline of the events necessary to implement the FNS hand system with SP. 
caused by the inactivity of the second immobilization period, the muscles were exercised by stimulation for 2 weeks before grasp patterns were created. After this exercise period, stimulated responses were fused and strong.

In Figure 5, the stimulation patterns are represented graphically with respect to shoulder position. For both grasps, movement of the contralateral shoulder from depression, through neutral, to elevation stimulates the hand to full prehensile strength. Pulse durations are chosen to maximize primary muscle force without secondary muscle recruitment. As seen in Figure 5a, for the lateral grasp the finger flexor muscles are first recruited to their maximum pulse durations, followed by stimulation of the thumb flexors and adductor. The flexed fingers form a base against which the thumb applies pinch force. For the palmar grasp, Figure 5b, recruitment of the thumb abductor is constant throughout the grasp while the finger flexors are ramped to their maximum pulse durations. ${ }^{5}$ For both grasps, voluntary finger and thumb extension to acquire an object is used.
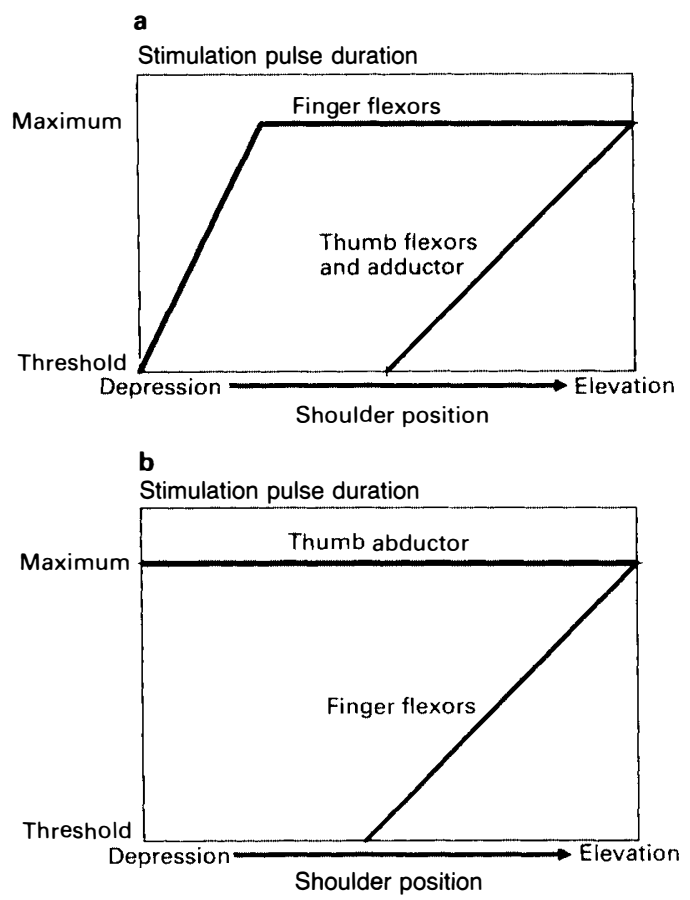

Figure 5 Lateral (a) and palmar (b) stimulation patterns as a function of shoulder position. Movement of the contralateral shoulder from depression, through neutral, to elevation stimulates the grasps to full prehensile strength.

\section{System training}

Training was separated into 3 different stages, each reflecting aspects of system operation: system mode recognition, shoulder transducer training, and coordination of voluntary grasp extension with stimulated grasp flexion.

To learn the audio cues that indicate the system modes (both grasps, lock, and on/off), the shoulder transducer was donned with stimulation deactivated. The boy was asked to activate different system modes by depressing the control switch attached to the shoulder transducer and listening for the audio cues. $\mathrm{He}$ mastered the relationship between the audio cues and system modes after 2 20-minute sessions.

To introduce him to the shoulder transducer, a training tool was devised which allowed it to operate a radio-controlled car. After several sessions using the transducer with the car, proximal muscle control appeared to improve in fine coordination and he learned the necessary shoulder movements. ${ }^{13}$

In the last stage, he was asked to coordinate his voluntary grasp extension with stimulated grasp flexion by gradually extending his fingers and thumb as he visually and cutaneously sensed a decrease in stimulation to his finger and thumb flexors. Similarly, as stimulation to his flexors increased, he was asked to relax his extensors. He practiced coordinating his grasps by prehending the objects used in the hand assessment and utensils, pencils, and soda cans.

By the end of the third month, he was able to coordinate his voluntary extension with stimulation and use the shoulder transducer to control his lateral and palmar grasps.

\section{Results and discussion}

Functional benefits of the FNS hand system were evaluated using manual muscle tests and CWRU's hand function assessment, by observing ADL performance with and without the hand system, and through telephone interviews with the patient and his family. All data were collected by the research occupational therapist.

\section{Manual muscle tests}

The results of 4 manual muscle tests are depicted in Table II. The first muscle test (Test 1) was performed during the screening process prior to FNS implementation. Test 2 was performed after the initial exercise 
period while Tests 3 and 4 occurred approximately 6 months and one year after the first test, respectively. Table II shows that increases in strength were observed in both stimulated and non stimulated muscles of the left upper extremity.

After the initial exercise period (Test 2), the strength of the extensor digitorum communis (EDC) and lumbricales increased to grade 4 . By Test 3, the strength of the abductor pollicis longus (AbPL) muscle increased by 2 muscle grades to grade 5 . Even though they were not stimulated, these muscles may have been strengthened when the boy extended his fingers and thumb against stimulated flexion.

For muscles that were stimulated, increases in volitional muscle strength from grade 1 to 2 were observed in the FDS of the long and ring finger by Test 2 and FDP of the ring finger by Test 4 .

As a result of increased strength in his EDC muscle of the left hand, he is now classified as an $\mathrm{O}(\mathrm{CU}): 6$. There were no changes in strength in the right forearm and hand musculature, suggesting that increases in muscles of the left hand were not due to maturity.

\section{Hand function assessment}

Five evaluation sessions were performed, 2 prior to, and 3 following home use of the FNS system. The Wilcoxon rank-sum test was used to determine if inconsistencies existed in the boy's performance. The null hypothesis is that the most recent sessions are not significantly different from previous sessions. A paired sign test was used to compare performance with and without the FNS system using the null hypothesis that there was no difference between them. For each object, the differences between FNS and volitional performance were computed for attempts, completions, and failures and compared to a hypothesized difference of zero. For both tests, the null hypothesis was rejected at the 95 percent confidence level.

\section{Consistency over time}

The performance in the first 2 sessions was compared to the last 3 in an effort to discern if functional use and exercise at home affected FNS and volitional performance.
During non FNS performance over the last 3 sessions, he made significantly fewer attempts $(p=0.001)$, completions $(p=$ $0.001)$ and failures $(p=0.019)$ with the block and fewer attempts $(p=0.001)$ and completions $(p=0.001)$ with the peg. Also, he had significantly fewer failures $(p=0.014)$ with cans during the later sessions. For all 5 sessions, he could not voluntarily manipulate the fork, weight, or videotape.

Because he could easily manipulate the peg and block, it appeared that these tasks were not challenging and did not keep his attention in the later sessions, causing a decrease in attempts and completions. The decrease in can and block failures may perhaps be attributed to the improved voluntary muscle grades of the EDC, APBL, lumbricales, and FDS of the long and ring fingers (Table II). Improved finger and thumb extension may have allowed him to more easily acquire the can and block while the increased strength in the lumbricales and finger flexors may have aided tenodesis function.

With the FNS system, there was no significant difference in the number of attempts, failures, or completions between the 2 groups of sessions for the weight, fork, tape, can, or block. For the peg, there was a significant increase in completions $(p=$ $0.021)$ and decrease in failures $(p=0.040)$, perhaps due to improved control of prehension with daily use of the system.

\section{FNS versus non FNS performance}

Analyses were performed separately for the first 2 and last 3 sessions and over all 5 sessions. For all objects, the observed significant differences were consistent across the session groupings.

Table III shows the results of the paired sign test over 5 sessions. For the peg, block, and can the 5-session median difference per trial in the number of attempts, completions, and failures is shown with the associated probability that the observed difference is equal to zero. For the weight, fork, and tape, objects which could only be prehended using the system, the median values with FNS are shown.

Without the hand system, the boy made 
Table III For the peg, block, and can, the 5-session median difference per trial between FNS and volitional performance is shown, with the probability that the observed difference is zero in parentheses. For the weight, fork, and tape, objects which could not be manipulated volitionally, the median number of attempts, completions, and failures per trial with FNS is presented. Negative medians indicate that greater numbers were recorded without FNS.

\begin{tabular}{|c|c|c|c|}
\hline Object & Attempts & Completions & Failures \\
\hline Peg & $-7(<.001)$ & $-9(<.001)$ & $1 \quad(.031)$ \\
\hline Block & $-12(<.001)$ & $-12(<.001)$ & $-1 \quad(.096)$ \\
\hline Can & $2(.210)$ & $2(.023)$ & $-2(<.001)$ \\
\hline Weight & 16 & 14 & 1 \\
\hline Fork & 11 & 10 & 0 \\
\hline Tape & 13 & 10 & 1 \\
\hline
\end{tabular}

significantly more attempts and completions with the block and peg and had fewer peg failures. It appeared that the volitional performance was better with these objects for 2 reasons. Occasionally, the shoulder transducer locked inadvertently as he tried to move the objects quickly, reducing attempts and completions with the system and causing failures. Also, both objects are light and small which allowed him to complete quickly and efficiently the tasks with his weak tenodesis.

In contrast, the can, which is heavier and wider, required a stronger tenodesis than the child consistently demonstrated. Stimulated prehension was stronger than his tenodesis and allowed SP to complete significantly more FNS can tasks with fewer failures.

The most difficult tasks, manipulation of the weight, fork, and tape, could not be performed volitionally but were completed with the hand system on a consistent basis with few failures. Table III shows that the median number of completions per trial for the weight, fork, and tape were 14,10 , and 10 respectively, with only 1,0 , and 1 median failures, respectively.

\section{Pinch force measurements}

Five measurements of stimulated lateral, palmar, and 5-finger pinch forces were taken before each session. Measurements were made with an adapted pinchometer with all attempts made to maintain the arm in adduction, elbow in 90 degrees and forearm and wrist in neutral. Table IV
Table IV Mean stimulated grasp force $(\mathrm{Kg})$ for the lateral, palmar and 5-finger pinch grasps calculated for each session. Standard deviations are shown in parentheses.

\begin{tabular}{lrrr}
\hline Session & Lateral & Palmar & \multicolumn{1}{c}{ 5-finger } \\
\hline 1 & $.4(.1)$ & $.3(0)$ & $.5(.1)$ \\
2 & $.3(0)$ & $.3(0)$ & $.3(0)$ \\
3 & $1.2(.2)$ & $.7(.1)$ & $1.1(.2)$ \\
4 & $.9(.2)$ & $.3(.1)$ & $.3(0)$ \\
5 & $.8(.3)$ & $.6(0)$ & $.7(.1)$ \\
\hline
\end{tabular}

shows the mean pinch forces with standard deviations. Generally, pinch forces were greater in the later 3 sessions. For each session, grasp forces were sufficient to manipulate all objects used in the hand function assessment.

\section{$A D L$ performance}

Among activities that have been compared thus far with the subject are eating with a fork, writing with a pencil, coloring with crayons, and painting with a brush. These activities were chosen because they were identified as those that were performed daily at home or school and required improved prehension.

He was able to perform all activities with and without FNS. However, 3 observations indicated that performance with FNS was more desirable than his typical bimanual performance. First, during writing, coloring, and painting, the unilateral function achieved with FNS enabled the boy to use 
the contralateral upper extremity to stabilize papers or coloring books which appeared to improve the quality of writing and coloring (Fig 6). Second, during all activities with FNS he was able to use his contralateral upper extremity to stabilize himself in his wheelchair which enhanced proximal stability and facilitated controlled unilateral distal function. The third observation was that he had less overall difficulty performing the activities while using the FNS hand system because of the greater force of the stimulated grasps, the ability to perform bilateral activities, and the expanded work area (Fig $6)$.

\section{Telephone interviews}

Over a one year period, weekly telephone interviews were conducted with the child and his parents to log daily system usage and to record their perceptions of the FNS system. During these interviews, his mother reported consistent use of the hand system at school for writing and handling papers and at home for eating. She reported an overall increase in self esteem and confidence while using the system. The boy and his family appeared to have integrated the maintenance and donning and doffing of the hand system into their daily routines and also integrated quarterly visits to Philadelphia into their schedules.

\section{Electrode survival}

A total of 31 electrodes were implanted: 14 thumb flexors, 10 thumb abductors, and 7 finger flexors. Thirteen electrodes have failed: 8 due to breakage and 5 due to adverse sensation.

Electrode breakage may be due to several factors. His voluntary wrist motion and finger extension (Tables I and II) may stress electrodes crossing the wrist. Stress may also be created by voluntary pronation and supination as well as stimulated contractions of other forearm muscles. Muscle hypertrophy may also play a significant role. Eight breakages occurred among the 17 initial electrodes while none have occurred among 14 electrodes implanted after the first 6 weeks of exercise.

Of the 5 electrodes that were removed

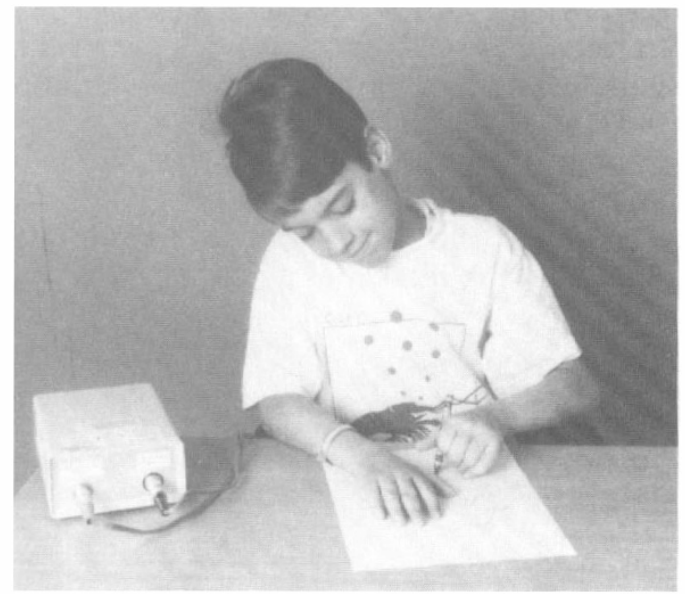

Figure 6 SP's lateral prehension with FNS. Bilateral function is achieved. During writing activities with FNS, SP's trunk stability is significantly improved.

because of adverse sensation, one was implanted in the FPL and 2 each in the FPB and AdP muscles. Upon stimulation of these electrodes, the child reported a subcutaneous discomfort along the radial border of the dorsum of the forearm. Researchers at CWRU have reported that a C6 subject experienced similar sensations upon stimulation of the FPL muscle. ${ }^{15}$

\section{Conclusion}

A neuroprosthetic hand system designed for adults with spinal cord injuries at the $\mathrm{C} 5$ and C6 level has been successfully modified for use by a child with function at the $\mathrm{C} 7$ level. Functional benefits of the hand system have been realized in the home and school environments. The child approaches self care, play, and academic activities with greater ease and less effort. The hand system frees his contralateral upper extremity for either postural balance, vastly expanding his work area, or stabilization of an object for use with the stimulated grasp, allowing finer hand control during ADLs.

This study shows that a young child can successfully participate in electrode implantation and electrode characterization, and can use the contralateral shoulder to coordinate stimulation with existing muscle function. Stimulated exercise strengthens 
stimulated muscles and may result indirectly in increased strength of non stimulated muscles, enhancing volitional grasps.

This work suggests that FNS has applications to individuals with low cervical level injuries and that children possess the faculties needed to successfully integrate the system into spontaneous activities.

\section{Acknowledgements}

This project is supported by Shriners Hospitals for Crippled Children Grant 15954 in collabora- tion with Case Western Reserve University. The authors would like to acknowledge $P$ Hunter Peckham PhD and the staff at CWRU for their ongoing support and advice on this project, Albert Weiss MD for sharing his expertise in hand rehabilitation, Gail Huss RN and Ellen Jantzen MD for their ongoing collaboration, and Kathy Goodstein for taking the photographs for this manuscript. Special gratitude is extended to the patient, his parents and brother for their dedication and commitment to this research program. Special consent has been given by the child's mother for inclusion of the photographs in this paper.

\section{References}

1 Mulcahey MJ, Smith BT, Triolo RJ, Betz RR (1991) Restoration of hand function in the C7 spinal cord injured child. J Am Paraplegia Soc 14 (2): 101-102.

2 Peckham PH, Mortimer JT (1978) Restoration of hand function in the quadriplegic through electrical stimulation. In: Reswick JB, Hambrecht FT, editors. Functional Electrical Stimulation: Applications in Neural Prosthesis. Marcel Dekker Inc, New York: 83-95.

3 Peckham PH, Mortimer JT, Marsolais EB (1980) Controlled prehension and release in the C5 quadriplegic elicited by functional electrical stimulation of the paralyzed forearm musculature. Annals Biomed Eng 8: 396-388.

4 Peckham PH, Marsolais EB, Mortimer JT (1980) Restoration of key grip and release in the C6 quadriplegic through functional electrical stimulation. J Hand Surg 5 (5): 462-469.

5 Kilgore KL, Peckham PH, Thrope GB, Keith MW, Gallaher-Stone KA (1989) Synthesis of hand grasp using functional neuromuscular stimulation. IEEE Trans Biomed Eng 36: 761-770.

6 Buckett JR, Peckham PH, Thrope GB, Braswell SD, Keith MW (1988) A flexible, portable system for neuromuscular stimulation in the upper extremity. IEEE Trans Biomed Eng 35: 897-904.

7 Wijam C, Stroh K, Van Doren C (1990) Functional evaluation of quadriplegic patients using a hand neuroprosthesis system. Arch Phys Med Rehabil 71: 1053-1057.

8 Thrope GB, Stroh KC, Crago PE, Peckham PH, Van Doren C (1988) Quantitative assessment of object acquisition and release using a neural prosthetic hand system with $\mathrm{C} 5$ and $\mathrm{C} 6$ quadriplegics. Proceedings of ICAART 88-Montreal: 354-355.

9 Seddon HJ (1972) In: Surgical Disorders of the Peripheral Nerves. Williams and Wilkins, Baltimore.

10 Bell-Krotoski J, Breger D, Beach R (1990) Applications of biomechanics for evaluation of the hand. In: Rehabilitation of the Hand: Surgery and Therapy. 3rd ed. CV Mosby Co, Philadelphia: 139-166.

11 Moberg E (1990) Surgical Rehabilitation of the Upper Limb in Tetraplegia. Paraplegia 28: 330-34.

12 Stroh K, Thrope G, Peckham PH, Keith MW, Menger V (1988) Candidate selection for use with a functional neuromuscular stimulation hand system. Proceedings of ICAART 88-Montreal: 330-331.

13 Smith BT, Mulcahey MJ, Triolo RT, and Betz RR (1991) Training tools for a neuroprosthetic hand system. Proceedings of the RESNA 14th Annual Conference: 394-396.

14 Stroh K, Thrope G, Peckham PH (1991) Personal communication. 